\title{
The BBGKY Hierarchy in Quantum Statistical Mechanics *
}

\author{
Sezar Fesciyan \\ Belfer Graduate School of Science, Yeshiva University, New York, USA
}

Received September 11, 1972

\begin{abstract}
It is shown that the infinite volume limit of the equilibrium reduced density matrices, shown by Ginibre to exist at low densities, satisfy the quantum time independent BBGKY hierarchy of equations. This extends analogous results for classical systems due to Gallavotti.
\end{abstract}

\section{Introduction}

Gallavotti [1] has shown rigorously that the infinite volume equilibrium correlation functions for a classical system satisfy the time independent BBGKY hierarchy of equations for a large class of potentials whenever the activity is small enough. For this purpose he made use of results due to Groeneveld, Penrose and Ruelle (see Chapter 4 in [2]) relating to the existence of the correlation functions in the thermodynamic limit. We made use of the corresponding results due to Ginibre $[3,4]$ relating to the existence of the quantum counterparts of the correlation functions, namely the reduced density matrices (RDM), and a method suggested by Gallavotti to prove that the time independent form of the quantum version of the BBGKY are satisfied by the infinite volume RDM. We treat the two cases of classical Boltzmann Statistics (C.S.) and Quantum Statistics (Q.S.), both Bose and Fermi, separately.

We are in general interested in a grand ensemble of systems of identical particles, not necessarily in equilibrium, enclosed in a finite box $A$, and interaction via two-body forces so that the Hamiltonian of the system with $N$ particles in $\Lambda$ has the form

$$
H_{N}=-\sum_{1 \leqq i \leqq N} \nabla_{x_{i}}^{2}+\sum_{1 \leqq i<j \leqq N} \Phi\left(x_{i}-x_{j}\right)
$$

where $2 m=\hbar=1, x_{\imath} \in \Lambda \subset R^{v}, \nabla_{x_{i}} \equiv \frac{\partial}{\partial x_{i}}$ and $v$ is the dimensionality of space.

* Supported by AFOSR Contract Number F 44620-71-C-0013. 
In the coordinate representation the nonequilibrium RDM can be defined by

$$
\varrho^{\Lambda}\left(x^{n} ; y^{n}, t\right)=\sum_{N=n}^{\infty} P_{N} \varrho_{n}^{N, \Lambda}\left(x^{n} ; y^{n}, t\right)
$$

with $P_{N}$ the time independent probability for the system to have $N$ particles where $x^{n} \equiv\left(x_{1}, \ldots, x_{n}\right)$, the superscript $\Lambda$ makes it explicit that the system is enclosed in a finite box, $\varrho_{n}^{N, A}\left(x^{n} ; y^{n}, t\right)$ are RDM suitable for an ensemble of similar systems of fixed $N$ and are obtained from a density matrix $\varrho^{N, \Lambda}\left(x^{N} ; y^{N}, t\right)$ via the relation

$$
\varrho_{n}^{N, \Lambda}\left(x^{n} ; y^{n}, t\right)=\frac{N !}{(N-n) !} \operatorname{Tr}^{(N-n)} \varrho^{N, \Lambda}\left(x^{N} ; y^{N}, t\right)
$$

with $\operatorname{Tr}^{(N)} \varrho^{N, A}\left(x^{N} ; y^{N}, t\right)=1$. Of course given a set of $\varrho_{n}^{N, A}\left(x^{n} ; y^{n}, t\right)$ Eq. (1.2) will not in general exist even for finite $A$. We suppose this not to be the case, i.e. coefficients $P_{N}$ and the $\varrho_{n}^{N, A}$ are such that (1.2) makes sense. Then the BBGKY equations satisfied by $\varrho^{\Lambda}\left(x^{n} ; y^{n}, t\right)$ are easily obtained from those satisfied by $\varrho_{n}^{N, A}\left(x^{n} ; y^{n}, t\right)$, the latter being obtained via successive trace taking on the Liouville equation satisfied by $\varrho^{N, \Lambda}\left(x^{N} ; y^{N}, t\right)$ (see Bogoliubov [5] for a derivation of this). The result is

$$
\begin{aligned}
& i \frac{\partial}{\partial t} \varrho^{\Lambda}\left(x^{n} ; y^{n}, t\right)=-\sum_{1 \leqq i \leqq n}\left(\nabla_{x_{i}}^{2}-\nabla_{y_{i}}^{2}\right) \varrho^{\Lambda}\left(x^{n} ; y^{n}, t\right) \\
& \quad+\left[\sum_{1 \leqq i<j \leqq n} \Phi\left(x_{i}-x_{j}\right)-\Phi\left(y_{i}-y_{j}\right)\right] \varrho^{\Lambda}\left(x^{n} ; y^{n}, t\right) \\
& \quad+\int d x_{n+1}\left[\Sigma \Phi\left(x_{i}-x_{n+1}\right)-\Phi\left(y_{i}-x_{n+1}\right)\right] \varrho^{\Lambda}\left(x^{n}, x_{n+1} ; y^{n}, x_{n+1}, t\right) .
\end{aligned}
$$

In the Q.S. case this equation is more readily obtained in a second quantized formalism using the equivalent definition of in terms of creation and annihilation operators:

$$
\varrho^{\Lambda}\left(x^{n} ; y^{n}, t\right)=\left\langle a^{+}\left(y^{n}, t\right) a\left(x^{\bar{n}}, t\right)\right\rangle \text { Thermal Average }
$$

where $a^{+}\left(y^{n}\right)=a^{+}\left(y_{1}\right) \ldots a^{+}\left(y_{n}\right), a\left(x^{\bar{n}}\right)=a\left(x_{n}\right) \ldots a\left(x_{1}\right)$ and $a^{+}(x)$ and $a(x)$ satisfy the well known commutation and anticommutation relations for Bose-Einstein and Fermi-Dirac statistics respectively. Then using the equations of motion

$$
i \frac{\partial}{\partial t} a^{+}(x, t)=\left[a^{+}(x, t), H^{\Lambda}\right]
$$

one obtains directly the Eq. (1.3) above.

At the present time very little is known about the existence of solutions to Eq. (1.3) in the infinite volume limit. If however, we restrict ourselves to the question of stationary solutions we can show that the infinite 
volume limit of the equilibrium RDM in fact satisfy the above equation, within the left side equal to zero; and this is what is done in the following pages. The stationary BBGKY are given by

$$
\begin{aligned}
0= & -\sum_{1 \leqq i \leqq n}\left(\nabla_{x_{i}}^{2}-\nabla_{y_{i}}^{2}\right) \varrho^{\Lambda}\left(x^{n} ; y^{n}\right) \\
& +\left[\sum_{1 \leqq i<j \leqq n} \Phi\left(x_{i}-x_{j}\right)-\Phi\left(y_{i}-y_{j}\right)\right] \varrho^{A}\left(x^{n} ; y^{n}\right) \\
& +\int d x_{n+1}\left[\sum_{1 \leqq i \leqq n} \Phi\left(x_{i}-x_{n+1}\right)-\Phi\left(y_{i}-x_{n+1}\right)\right] \varrho^{\Lambda}\left(x^{n}, x_{n+1} ; y^{n}, x_{n+1}\right) .
\end{aligned}
$$

We will show that every term in Eq. (1.4) makes sense in the infinite volume limit and furthermore that the equality in Eq. (1.4) persists when $\Lambda \rightarrow \infty$. The sense in which the limit $\Lambda \rightarrow \infty$ is to be understood is that the coordinates, $\left(x^{n}\right)$ and $\left(y^{n}\right)$ stay within an arbitrary compact set. We make essential use of the result, obtained by Ginibre [3] and the formalism developed by him for the quantum RDM. He shows that $\varrho\left(x^{n} ; y^{n}\right)$, the infinite volume limits of the equilibrium RDM, exist, are bounded and $\varrho^{\Lambda}\left(x^{n} ; y^{n}\right) \rightarrow \varrho\left(x^{n} ; y^{n}\right)$ uniformly on compact sets, provided one is restricted to the class of potentials such that
a) $\Phi(x, y) \equiv \Phi(x-y)$ is continuous
b) $\int_{\text {all space }}|\Phi(x)| d x<\infty$ (integrability)
c) $\sum_{1 \leqq i<j \leqq n} \Phi\left(x_{i}-x_{j}\right) \geqq-n B$ (stability)

and furthermore one is restricted to a region in the complex activity plane whose radius is determined by the potential $\Phi(x)$ and the temperature $1 / \beta$. Working always within this restricted domain we shall see that we need to impose further conditions on the potentials $\Phi(x)$ so that Eq. (1.4) will hold in the thermodynamic limit.

With the above in mind it is easy to see that the last two terms in Eq. (1.4) exist in the $A \rightarrow \infty$ limit and that they are the uniform limits of the corresponding terms for finite $\Lambda$. For the last term we use the fact (see Ginibre [3]) that

$$
\left|\varrho^{A}\left(x^{n+1} ; y^{n+1}\right)\right| \leqq \frac{\xi^{n+1}}{\lambda^{v(n+1)}}\|\varrho\|_{\xi}, \quad \xi>0
$$

for Boltzmann statistics and

$$
\left|\varrho^{\Lambda}\left(x^{n+1} ; y^{n+1}\right)\right| \leqq \frac{(n+1) !}{\lambda^{v(n+1)}}\left[g_{v / 2}(\xi)\right]^{n+1}\|\varrho\|_{\xi}, \quad 0<\xi<1
$$

for Quantum statistics, where $\lambda=\sqrt{\pi \beta}$ is the thermal wavelength, and $\|\varrho\|_{\xi}$ is the norm of a sequence of functions $\left\{\varrho\left(\omega^{n}\right)\right\}$ regarded as a vector 
belonging to a suitable Banach space. The nature of $\varrho\left(\omega^{n}\right)$ and its relationship to $\varrho\left(x^{n} ; y^{n}\right)$ will be clearer later on (also see Ginibre [6]). We see now that in the infinite volume limit the integral

$$
\int d x_{n+1}\left[\sum_{1 \leqq i \leqq n} \Phi\left(x_{i}-x_{n+1}\right)-\Phi\left(y_{i}-x_{n+1}\right)\right] \varrho\left(x^{n}, x_{n+1} ; y^{n}, x_{n+1}\right)
$$

exists due to the bounds (1.6) or (1.7) and the integrability condition (1.5b). Furthermore the corresponding term for finite $\Lambda$ approaches to the above uniformly in $\left(x^{n}, y^{n}\right)$.

The main problem is to show that the infinite volume RDM have derivatives with respect to their arguments. From here on we will treat the two cases of classical and quantum statistics separately, the latter being algebraically more involved. We are allowed to consider classical (Boltzmann) statistics in a quantum formalism because there is no "correct" statistics that is logically imposed by the theory within the nonrelativistic domain.

\section{Classical Statistics:}

We make use of a representation of the RDM as a Wiener integral of a functional $\varrho\left(\omega^{n}\right)$. In the case of C.S. this representation has the form

$$
\varrho\left(x^{n} ; y^{n}\right)=\int P_{x^{n}, y^{n}}^{\beta}\left(d \omega^{n}\right) \varrho\left(\omega^{n}\right)
$$

(see Ginibre [6], Kac [7]), where

$$
\begin{array}{rll}
x^{n} \equiv\left(x_{1}, \ldots, x_{n}\right) & \text { and } & x_{j} \in R^{v} \\
\omega^{n} \equiv\left(\omega_{1}, \ldots, \omega_{n}\right) & \text { and } & \omega_{j} \text { is a map } \\
\omega_{j}:[0, \beta] \rightarrow R^{v} \ni \omega_{j}(0)=x_{j} & \text { and } & \omega_{j}(\beta)=y_{j}
\end{array}
$$

in fact a Brownian trajectory. $P_{x^{n}, y^{n}}^{\beta}\left(d \omega^{n}\right)$ is the conditional Wiener measure defined on the space of such trajectories. The exact form of $\varrho\left(\omega^{n}\right)$ is immaterial for our purposes (see [6]). Equation (2.1) can also be written as

$$
\varrho\left(x^{n} ; y^{n}\right)=\left[\frac{e^{-\sum_{J=1}^{n}\left(x_{J}-y_{J}\right)^{2} ! \beta}}{\lambda^{n v}}\right] \int E_{x^{n}, y^{n}}^{\beta}\left(d \omega^{n}\right) \varrho\left(\omega^{n}\right)
$$

where the factor in brackets is a normalization and equals $\int P_{x^{n}, y^{n}}^{\beta}\left(d \omega^{n}\right)$, and $E_{x^{n}, y^{n}}^{\beta}\left(d \omega^{n}\right)$ is now the normalized conditional Wiener measure.

If we try to differentiate Eq. (2.2) with respect to some $x_{\alpha}$ we get involved in differentiating the Wiener measure which depends on the end points of $\omega_{\alpha}$. We can avoid this by a change of variables over which we integrate. Every trajectory $\omega$ that starts at $x$ and ends at $y$ can be uniquely 
obtained by adding to the fixed straight line path from $x$ to $y$ a trajectory $\omega^{\prime}$ that starts and ends at the origin. That is,

with

$$
\omega(t)=\omega^{\prime}(t)+\mu(t) \text { for all } 0 \leqq t \leqq \beta
$$

$$
\mu(t)=\frac{t}{\beta} y+\left(1-\frac{t}{\beta}\right) x,
$$

the fixed straight line path in $R^{v}$. Then we have

$$
\varrho\left(x^{n} ; y^{n}\right)=\left[\frac{e^{-\sum\left(x_{j}-y_{j}\right)^{2} / \beta}}{\lambda^{n v}}\right] \int E_{00}^{\beta}\left(d \omega^{\prime n}\right) \varrho\left(\left(\omega^{\prime}+\mu\right)^{n}\right) .
$$

Now differentiation is easy:

$$
\begin{aligned}
\frac{\partial \varrho\left(x^{n} ; y^{n}\right)}{\partial x_{\alpha}}= & \frac{\partial}{\partial x_{\alpha}}\left[\frac{e^{-\sum_{j}\left(x_{j}-y_{j}\right)^{2} / \beta}}{\lambda^{n v}}\right] \int E_{00}^{\beta}\left(d \omega^{\prime n}\right) \varrho\left(\omega^{\prime n}+\mu^{n}\right) \\
& \left.+\frac{e^{-\sum_{j}\left(x_{j}-y_{j}\right)^{2} / \beta}}{\lambda^{n v}}\right] \int E_{00}^{\beta}\left(d \omega^{\prime n}\right) \frac{\partial \varrho\left(\omega^{n}\right)}{\partial \mu_{\alpha}(0)}
\end{aligned}
$$

where we have denoted functional differentiation of $\varrho(\omega)$ with partial derivative symbols. So the problem comes down to showing that $\varrho\left(\omega^{n}\right)$ are functionally differentiable [the above argument is due to H. P. McKean]. (Private communication via Gallavotti). The set of Mayer-Montroll equations or the set of Kirkwood-Salzburg equation (or rather the quantum analogue of these equations) can be used equally well for this purpose in the C.S. case. However in the Q.S. case the required differentiability can be shown to be true only for repulsive potentials if we use the M.M. equations. The K.S. equations on the other hand give the required result with no more restrictions on $\Phi(X)$ in the Q.S. case than are necessary in the C.S. case (I thank Dr. Ginibre for bringing this to my attention). So that we proceed to work with the K.S. equations. It is the following unsymmetrized form of the K.S. equations (see [6]) which is useful for our purposes:

$$
\begin{aligned}
& \varrho\left(\omega^{n}\right)=z e^{-W\left(\omega_{\alpha}, \omega^{n^{\prime}}\right)} \sum_{m=0}^{\infty} \frac{1}{m !} \int P_{u^{m}, u^{m}}^{\beta}\left(d \bar{\omega}^{m}\right) d u^{m} K\left(\omega_{\alpha}, \bar{\omega}^{m}\right) \varrho\left(\omega^{n^{\prime}}, \bar{\omega}^{m}\right) \\
& \begin{aligned}
W\left(\omega_{\alpha}, \omega^{n^{\prime}}\right) & =\sum_{\substack{j=1 \\
j \pm \alpha}}^{n} \varphi\left(\omega_{\alpha}-\omega_{j}\right) \\
K\left(\omega_{\alpha}, \bar{\omega}^{m}\right) & =\prod_{j=1}^{m}\left\{e^{-\varphi\left(\omega_{\alpha}-\bar{\omega}_{j}\right)}-1\right\} \\
\varphi(\omega) & \equiv \int_{0}^{\beta} \Phi[\omega(t)] d t
\end{aligned}
\end{aligned}
$$


Eq. (2.6) holds in the infinite volume limit and we can differentiate it to study the infinite volume derivatives. Denoting the functional derivatives $\frac{\delta}{\delta \omega_{\alpha}(0)}$ or $\frac{\delta}{\delta \omega_{\alpha}(\beta)}$ by the ordinary partial derivative symbols $\frac{\partial}{\partial \omega_{\alpha}}$ we have

$$
\begin{aligned}
& \frac{\partial \varrho\left(\omega^{n}\right)}{\partial \omega_{\alpha}}=z\left(-\sum_{\substack{j=1 \\
j \neq \alpha}}^{n} \frac{\partial \varphi\left(\omega_{j}-\omega_{\alpha}\right)}{\partial \omega_{\alpha}}\right) \\
& \cdot e^{-W\left(\omega_{\alpha}, \omega^{n^{\prime}}\right)} \sum_{m=0}^{\infty} \frac{1}{m !} \int P_{u^{m}, u^{m}}^{\beta}\left(d \bar{\omega}^{m}\right) d u^{m} K\left(\omega_{\alpha}, \bar{\omega}^{m}\right) \varrho\left(\omega^{n^{\prime}}, \bar{\omega}^{m}\right) \\
& \quad+z e^{-W\left(\omega_{\alpha}, \omega^{n^{\prime}}\right)} \sum_{m=0}^{\infty} \frac{1}{m !} \int P_{u^{m}, u^{m}}^{\beta}\left(d \bar{\omega}^{m}\right) d u^{m} \frac{\partial K\left(\omega_{\alpha}, \bar{\omega}^{m}\right)}{\partial \omega_{\alpha}} \varrho\left(\omega^{n^{\prime}}, \bar{\omega}^{m}\right)
\end{aligned}
$$

with

$$
\frac{\partial K\left(\omega_{\alpha}, \bar{\omega}^{m}\right)}{\partial \omega_{\alpha}}=\sum_{j=1}^{m}\left(\frac{\partial e^{-\varphi\left(\omega_{\alpha}-\bar{\omega}_{j}\right)}}{\partial \omega_{\alpha}}\right) \prod_{\substack{i=1 \\ i \neq j}}^{m}\left\{e^{-\varphi\left(\omega_{\alpha}-\bar{\omega}_{\nu}\right)}-1\right\}
$$

Equation (2.7) makes sense whenever the right hand side converges uniformly and we see that the first term already requires that the potential be differentiable with continuous derivative; continuity is required for later bounds.

Now consider

$$
\int P_{u^{m}, u^{m}}\left(d \bar{\omega}^{m}\right) d u^{m}\left|\sum_{j=1}^{m}\left(\frac{\partial e^{-\varphi\left(\omega_{\alpha}-\bar{\omega}_{j}\right)}}{\partial \omega_{\alpha}}\right) \prod_{\substack{i=1 \\ i \neq j}}^{m}\left\{e^{-\varphi\left(\omega_{\alpha}-\bar{\omega}_{i}\right)}-1\right\} \varrho\left(\omega^{n^{\prime}}, \bar{\omega}^{m}\right)\right|
$$

using $\left|\varrho\left(\omega^{n^{\prime}}, \bar{\omega}^{m}\right)\right| \leqq \xi^{m+n-1}\|\varrho\|$.

Eq. (2.8) is

and since

$$
\begin{gathered}
\leqq \xi^{m+n-1}\|\varrho\| \sum_{j=1}^{m}\left(\prod_{\substack{i=1 \\
i \neq j}}^{m} \int P_{u_{i} u_{i}}\left(d \bar{\omega}_{i}\right) d u_{i}\left|K\left(\omega_{\alpha}, \bar{\omega}_{i}\right)\right|\right) \\
\cdot\left(\int P_{u_{j} u_{j}}\left(d \bar{\omega}_{j}\right) d u_{j} \mid \frac{\partial e^{-\varphi\left(\omega_{\alpha}-\bar{\omega}_{J}\right)}}{\partial \omega_{\alpha}} \|\right)
\end{gathered}
$$

where

$$
\int P_{u u}(d \bar{\omega}) d u|K(\omega, \bar{\omega})| \leqq C(\beta)
$$

$$
C(\beta) \equiv \frac{\beta}{\lambda^{v}}\left(e^{\beta b} \int \Phi_{-}(x) d x+\int \Phi_{+}(x) d x\right)
$$

and $\Phi_{ \pm}(x)=\max ( \pm \Phi(x), 0)$ (see $\left.[6]\right)$.

Eq. (2.8) is

$$
\leqq \xi^{m+n-1}\|\varrho\| m[C(\beta)]^{m-1} \int P_{u u}(d \bar{\omega}) d u\left|\frac{\partial e^{-\varphi\left(\omega_{\alpha}-\bar{\omega}\right)}}{\partial \omega_{\alpha}}\right| .
$$


Now

$$
\int d u \int P_{u u}(d \bar{\omega})\left|\frac{\partial e^{-\varphi\left(\omega_{\alpha}-\bar{\omega}\right)}}{\partial \omega_{\alpha}}\right|=\int P_{00}\left(d \omega^{\prime}\right) \int d u\left|\frac{\partial e^{-\varphi\left(\omega_{\alpha}-\omega^{\prime}-u\right)}}{\partial \omega_{\alpha}}\right|
$$

(where a simple translation in $\bar{\omega}$ has been made).

$$
\leqq \sup _{\omega, \omega^{\prime}}\left[\int d u\left|\frac{\partial e^{-\varphi\left(\omega_{\alpha}-\omega^{\prime}-u\right)}}{\partial \omega_{\alpha}}\right|\right] \cdot \int P_{00}\left(d \omega^{\prime}\right)=\frac{1}{\lambda^{v}} \int d x\left|\frac{\partial e^{-\beta \Phi(x)}}{\partial x}\right| .
$$

We see that the required convergence will come about when we restrict the class of admissible potentials further by the following condition:

$$
D(\beta) \equiv \frac{1}{\lambda^{v}} \int d x\left|\frac{\partial e^{-\beta \Phi(x)}}{\partial x}\right|<+\infty .
$$

Then the second series in (2.7) uniformly converges since we have

$$
\xi^{n}\|\varrho\| D(\beta) \sum_{m=0}^{\infty} \frac{1}{m !} \xi^{m-1} m[C(\beta)]^{m-1}=\xi^{n}\|\varrho\| D(\beta) \exp [\xi C(\beta)]
$$

with the above and the fact that

$$
\left|\frac{\partial e^{-W\left(\omega_{\alpha}, \omega^{n^{\prime}}\right)}}{\partial \omega_{\alpha}}\right| \leqq(n-1) \bar{\Phi} e^{-W\left(\omega_{\alpha}, \omega^{n^{\prime}}\right)}
$$

which comes about because $\left(\omega^{n}\right)$ are to lie within a compact set $\Lambda_{R} \subset \Lambda$ and we have assumed the continuity of $\frac{\partial \Phi(x)}{\partial x}(\bar{\Phi}$ is a uniform bound for $\frac{\partial \Phi}{\partial x}$ in $\left.\Lambda_{R}\right)$. Then

$$
\left|\frac{\partial \varrho\left(\omega^{n}\right)}{\partial \omega_{\alpha}}\right| \leqq(n-1) \bar{\Phi} \xi^{n}\|\varrho\|+z \xi^{n} e^{(n-1) \beta b}\|\varrho\| D(\beta) \exp [\xi C(\beta)]
$$

and referring back to Eq. (2.5) we see that $\frac{\partial \varrho\left(x^{n} ; y^{n}\right)}{\partial x_{\alpha}}$ or $\frac{\partial \varrho\left(x^{n} ; y^{n}\right)}{\partial y_{\alpha}}$ exist in the $\Lambda \rightarrow \infty$ limit.

To show the same result about the second derivatives which are relevant for Eq. (1.4) we demand similarly that the second order functional derivatives exist. This involves

$$
\begin{aligned}
& \frac{\partial^{2} K\left(\omega_{\alpha}, \bar{\omega}^{m}\right)}{\partial \omega_{\alpha}^{2}}=\sum_{j=1}^{m}\left(\frac{\partial^{2} e^{-\varphi\left(\omega_{\alpha}-\omega_{J}\right)}}{\partial \omega_{\alpha}^{2}}\right) K\left(\omega_{\alpha}, \bar{\omega}^{m^{\prime}}\right) \\
& \quad+\sum_{j=1}^{m} \sum_{\substack{i=1 \\
i \neq j}}^{m}\left(\frac{\partial e^{-\varphi\left(\omega_{\alpha}-\bar{\omega}_{J}\right)}}{\partial \omega_{\alpha}}\right)\left(\frac{\partial e^{-\varphi\left(\omega_{\alpha}-\bar{\omega}_{i}\right)}}{\partial \omega_{\alpha}}\right) \prod_{\substack{k=1 \\
k \neq i \\
k \neq j}}^{m}\left\{e^{-\varphi\left(\omega_{\alpha}-\bar{\omega}_{k}\right)}-1\right\}
\end{aligned}
$$


which leads similarly to the additional condition on the class of admissible potentials, namely that

$$
\int d x\left|\frac{\partial^{2} e^{-\beta \Phi(x)}}{\partial x^{2}}\right|<+\infty .
$$

The fact that the finite volume derivatives approach the infinite volume ones whose existence was established above follows from the uniform boundedness in $A$ of the integrands appearing in Eq. (2.7) by integrable functions and that the integrands approach their infinite volume limits point-wise.

Thus the equality in Eq. (1.4) persists as $\Lambda \rightarrow \infty$.

\section{Quantum Statistics:}

In this case $\varrho\left(x^{n} ; y^{n}\right)$ is again given by a Wiener Integral of a functional $\varrho\left(\hat{\omega}^{n}\right)$ but now the set of trajectories over which one has to integrate do not have the fixed length $\beta$ (length referes to the range of the parameter $t$ ) as before but are composed of several elementary trajectories of length $\beta$; furthermore a trajectory starting at $x_{\imath}$ may not end at $y_{i}$ as before but at some $y_{k}, k \neq i$. And finally one has to sum over all such possible trajectories to obtain $\varrho\left(x^{n} ; y^{n}\right)$ as follows:

$$
\varrho\left(x^{n} ; y^{n}\right)=\sum_{\pi \in S_{n}} \varepsilon^{\pi} \sum_{\mu_{1}=1}^{\infty} \cdots \sum_{\mu_{n}=1}^{\infty} \varepsilon^{q-n} \int P_{x^{n}, \pi\left(y^{n}\right)}^{\mu^{n} \beta}\left(d \hat{\omega}_{n}\right) \varrho\left(\hat{\omega}^{n}\right)
$$

where $\mu_{i}$ is the number of elementary trajectories of which the composite trajectory $\hat{\omega}_{i}$ is composed, $q=\sum_{i=1}^{n} \mu_{i}, \pi$ ranges over the elements of $S_{n}$, the group of permutations of $n$ objects, $\varepsilon=-1$ for Fermi-Dirac and $\varepsilon=+1$ for Bose-Einstein Statistics. $\varepsilon^{\pi}$ gives the signature of a given permutation $\pi \in S_{n}$ (see [6]).

The same argument of McKean holds in this case also but here we need to make two different changes of variables depending upon whether differentiability with respect to the initial point or the end point of a composite trajectory is required. Let $x_{\alpha}$ be the initial point of the composite trajectory $\hat{\omega}_{\alpha}(t)$ of length $\mu_{\alpha} \beta$, and let $y_{k}$ be its end point, i.e. $y_{k}=\hat{\omega}_{\alpha}\left(\mu_{\alpha} \beta\right)$. To establish differentiability with respect to $x_{\alpha}$ we single out the first elementary trajectory $\omega_{\alpha}(t)$ starting at $x_{\alpha}$ and ending at $\hat{\omega}_{\alpha}(\beta)=u_{\alpha}$, the first intermediate point on $\hat{\omega}_{\alpha}(t)$. Now a change of variables $\omega_{\alpha}(t) \rightarrow \omega_{\alpha}^{\prime}(t)$ defined by

$$
\omega_{\alpha}(t)=\omega_{\alpha}^{\prime}(t)+\left(1-\frac{t}{\beta}\right)\left(x_{\alpha}-y_{k}\right)
$$


frees the Wiener measure in (3.1) from its $x_{\alpha}$ dependence as in the C.S. case above and the argument there goes through so that we have again: it is necessary that $\varrho\left(\hat{\omega}^{n}\right)$ be functionally differentiable for $\frac{\partial \varrho\left(x^{n} ; y^{n}\right)}{\partial x_{\alpha}}$ to exist. But one has to be more careful in this case since given the existence and boundedness of $\frac{\partial \varrho}{\partial \omega_{\alpha}}$, the infinite summations required to obtain $\frac{\partial \varrho\left(x^{n} ; y^{n}\right)}{\partial x_{\alpha}}$ may still not converge. It turns out in the following that this convergence actually occurs without any extra restrictions on the class of admissible potentials. This is the main advantage of using K.S. equations instead of the M.M. equations.

The K.S. equations have the following form ([6]):

$$
\begin{aligned}
\varrho\left(\hat{\omega}^{n}\right)= & z e^{-W\left(\omega_{\alpha}, \hat{\omega}^{n^{\prime}}\right)} \sum_{m=0}^{\infty} \frac{1}{m !} \sum_{j_{1}=1}^{\infty}\left(\frac{\varepsilon^{j_{1}-1}}{j_{1}}\right) \cdots \sum_{j_{m}=1}^{\infty}\left(\frac{\varepsilon^{j_{m}-1}}{j_{m}}\right) \\
& \cdot \int P_{u^{m}, u^{m}}^{j^{m} \beta}\left(d \bar{\omega}^{m}\right) d u^{m} K\left(\omega_{\alpha}, \bar{\omega}^{m}\right) \varrho\left(\hat{\omega}^{n^{\prime}}, \bar{\omega}^{m}\right) \quad\left(\left(\hat{\omega}^{n^{\prime}}\right)=\left(\hat{\omega}^{n}\right) \backslash \omega_{\alpha}\right)
\end{aligned}
$$

where $\omega_{\alpha}$ is the singled-out elementary trajectory discussed above,

$K\left(\omega_{\alpha}, \bar{\omega}^{m}\right)=\prod_{k=1}^{m}\left\{e^{-\varphi\left(\omega_{\alpha}-\bar{\omega}_{k}\right)}-1\right\}=\prod_{k=1}^{m}\left\{e^{-\sum_{l=0}^{J_{k}-1} j \Phi\left[\omega_{\alpha}(t)-\bar{\omega}_{k}(t+l \beta)\right] d t}-1\right\}(3.4)$ and

Differentiation gives

$$
W\left(\omega_{\alpha}, \hat{\omega}^{n^{\prime}}\right)=\sum_{\substack{k=1 \\ k \neq \alpha}}^{n} \sum_{l=0}^{\mu_{k}-1} \int_{0}^{\beta} \Phi\left[\omega_{\alpha}(t)-\hat{\omega}_{k}(t+l \beta)\right] d t
$$

$$
\begin{aligned}
\frac{\partial \varrho\left(\hat{\omega}^{n}\right)}{\partial \omega_{\alpha}}= & z\left(\frac{\partial e^{-W\left(\omega_{\alpha}, \hat{\omega} n^{\prime}\right)}}{\partial \omega_{\alpha}}\right) \sum_{m=0}^{\infty} \frac{1}{m !} \sum_{j_{1}=1}^{\infty}\left(\frac{\varepsilon^{j_{1}-1}}{j_{1}}\right) \cdots \sum_{j_{m}=1}^{\infty}\left(\frac{\varepsilon^{j_{m}-1}}{j_{m}}\right) \\
& \cdot \int P_{u^{m}, u^{m}}^{j^{m} \beta}\left(d \bar{\omega}^{m}\right) d u^{m} K\left(\omega_{\alpha}, \bar{\omega}^{m}\right) \varrho\left(\hat{\omega}^{n^{\prime}}, \bar{\omega}^{m}\right) \\
& +z e^{-W\left(\omega_{\alpha}, \hat{\omega} n^{\prime}\right)} \sum_{m=0}^{\infty} \frac{1}{m !} \sum_{j_{1}=1}^{\infty}\left(\frac{\varepsilon^{j_{1}-1}}{j_{1}}\right) \cdots \sum_{j_{m}=1}^{\infty}\left(\frac{\varepsilon^{j_{m}-1}}{j_{m}}\right) \\
& \cdot \int P_{u^{m}, u^{m}}^{j^{m} \beta}\left(d \bar{\omega}^{m}\right) d u^{m} \sum_{k=1}^{m}\left(\frac{\partial e^{-\varphi\left(\omega_{\alpha}-\bar{\omega}_{k}\right)}}{\partial \omega_{\alpha}}\right) K\left(\omega_{\alpha}, \bar{\omega}^{m^{\prime}}\right) \varrho\left(\hat{\omega}^{n}, \bar{\omega}^{m}\right),
\end{aligned}
$$

where $\frac{\partial}{\partial \omega_{\alpha}}$ means $\frac{\delta}{\delta \omega_{\alpha}(0)}$ and $m^{\prime}=(1, \ldots, k-1, k+1, \ldots, m)$. 
Eq. (3.5) converges uniformly as required since (see [6])

$$
\left|\sum_{j_{1}=1}^{\infty}\left(\frac{\varepsilon^{j_{1}-1}}{j_{1}}\right) \cdots \sum_{j_{m}=1}^{\infty}\left(\frac{\varepsilon^{j_{m}-1}}{j_{m}}\right) \int P_{u^{m}, u^{m}}^{j^{m} \beta}\left(d \bar{\omega}^{m}\right) d u^{m} K\left(\omega_{\alpha}, \bar{\omega}^{m}\right) \varrho\left(\hat{\omega}^{n^{\prime}}, \bar{\omega}^{m}\right)\right|
$$

is

$$
\begin{aligned}
& \leqq\|\varrho\| \xi^{q-1} \prod_{k=1}^{m} \sum_{j_{k}=1}^{\infty}\left(\frac{\xi^{j_{k}}}{j_{k}}\right) \int P_{u_{k} u_{k}}^{j_{k} \beta}\left(d \bar{\omega}_{k}\right) d u_{k}\left|K\left(\omega_{\alpha}, \bar{\omega}^{m}\right)\right| \\
& \leqq\|\varrho\| \xi^{q-1} \prod_{k=1}^{m}\left\{\sum_{j_{k}=1}^{\infty}\left(\frac{\xi^{j_{k}}}{j_{k}^{v / 2}}\right) \frac{\left(e^{\beta b}\right)^{j_{k}}}{\lambda^{v}} \int \Phi_{-}(x) d x\right. \\
& \left.+\sum_{j_{k}=1}^{\infty}\left(\frac{\xi^{j_{k}}}{j_{k}^{v / 2}}\right)\left(\frac{1}{\lambda^{v}}\right) \int \Phi_{+}(x) d x\right\} \\
& =\|\varrho\| \xi^{q-1}\left[\frac{\beta}{\lambda^{v}}\left\{g_{v / 2}\left(\xi e^{\beta b}\right) \int \Phi_{-}(x) d x+g_{v / 2}(\xi) \int \Phi_{+}(x) d x\right\}\right]^{m} \\
& \equiv\|\varrho\| \xi^{q-1}[\bar{C}(\beta, \xi)]^{m}
\end{aligned}
$$

and so the first series in (3.5) is bounded by the following convergent series

$$
\|\varrho\| \xi^{q-1} \sum_{m=0}^{\infty} \frac{1}{m !}[\bar{C}(\beta, \xi)]^{m} \equiv\|\varrho\| \xi^{q-1} \exp [\bar{C}(\beta, \xi)] .
$$

Similarly each term in the second series in (3.5) is bounded by

$$
\begin{aligned}
& \|\varrho\| \xi^{q-1} \frac{1}{m !} \sum_{k=1}^{m}\left(\prod_{\substack{i=1 \\
i \neq h}}^{m} \sum_{j_{i}=1}^{\infty}\left(\frac{\xi^{j_{2}}}{j_{i}}\right) \int P_{u_{i} u_{i}}^{j_{\beta} \beta}\left(d \bar{\omega}_{i}\right) d u_{i}\left|K\left(\omega_{\alpha}, \bar{\omega}_{i}\right)\right|\right) \\
& \cdot\left(\sum_{j_{k}=1}^{\infty}\left(\frac{\xi^{j_{k}}}{j_{k}}\right) \int P_{u_{k} u_{k}}^{j_{k} \beta}\left(d \bar{\omega}_{k}\right) d u_{k}\left|\frac{\partial e^{-\varphi\left(\omega_{\alpha}-\bar{\omega}_{k}\right)}}{\partial \omega_{\alpha}}\right|\right) \\
& \leqq\|\varrho\| \xi^{q-1} \cdot \frac{1}{m !} m[\bar{C}(\beta, \xi)]^{m-1} \sup _{\omega_{\alpha}} \sum_{j=1}^{\infty}\left(\frac{\xi^{j}}{j}\right) \int P_{u u}^{j \beta}(d \bar{\omega}) d u\left|\frac{\partial e^{-\varphi\left(\omega_{\alpha}-\bar{\omega}\right)}}{\partial \omega_{\alpha}}\right|
\end{aligned}
$$

Now,

$$
\begin{aligned}
\frac{\partial}{\partial \omega_{\alpha}} e^{-\varphi\left(\omega_{\alpha}-\bar{\omega}\right)} & \equiv \frac{\delta}{\delta \omega_{\alpha}(0)} \prod_{l=0}^{j-1} e^{-\int \Phi\left[\omega_{\alpha}(t)-\bar{\omega}(t+l \beta)\right] d t} \\
& =\sum_{l=0}^{j-1} \frac{\delta}{\delta\left(\omega_{\alpha}(0)\right.} e^{-\int_{0}^{\beta} \Phi\left[\omega_{\alpha}(t)-\bar{\omega}(t+l \beta)\right] d t} \prod_{\substack{l^{\prime}=0 \\
l^{\prime}=l}}^{-1} e^{-\int_{0}^{\beta} \Phi\left[\omega_{\alpha}(t)-\bar{\omega}\left(t+l^{\prime} \beta\right)\right] d t}
\end{aligned}
$$


and

$$
\begin{aligned}
& S P_{u u}^{j \beta}(d \bar{\omega}) d u\left|\frac{\partial e^{-\varphi\left(\omega_{\alpha}-\bar{\omega}\right)}}{\partial \omega_{\alpha}}\right| \\
& \leqq e^{(j-1) \beta b} \sup _{\omega_{\alpha}} \int P_{00}^{j \beta}\left(d \omega^{\prime}\right) \int d u \sum_{l=0}^{j-1}\left|\frac{\delta e^{-\int_{0}^{\beta} \Phi\left[\omega_{\alpha}(t)-\omega^{\prime}(t+l \beta)-u\right]}}{\delta \omega_{\alpha}(0)}\right| \\
& \leqq e^{(j-1) \beta b} j \sup _{\omega_{\alpha}} \sup _{\omega^{\prime} . l}\left[\int d u\left|\frac{\delta e^{-\int \Phi\left[\omega_{\alpha}(t)-\omega^{\prime}(t+l \beta)-u\right] d t}}{\delta \omega_{\alpha}(0)}\right|\right] \cdot \int P_{00}^{j \beta}\left(d \omega^{\prime}\right)
\end{aligned}
$$

then (3.7) is bounded by

$$
\begin{aligned}
& \|\varrho\| \xi^{q-1} \frac{1}{m !} m[\bar{C}(\beta, \xi)]^{m-1} e^{-\beta b}\left(\sum_{j} \frac{\xi^{j}}{j} \frac{j\left(e^{\beta b}\right)^{j}}{j^{v / 2}}\right) D(\beta) \\
= & \|\varrho\| \xi^{q-1} \frac{1}{m !} m e^{-\beta b}[\bar{C}(\beta, \xi)]^{m-1} D(\beta) g_{v_{k}}\left(\xi e^{\beta b}\right)
\end{aligned}
$$

furthermore

$$
\begin{aligned}
& \xi^{q-1}\|\varrho\| e^{-\beta b} D(\beta) g_{v / 2}\left(\xi e^{\beta b}\right) \sum_{m=0}^{\infty} \frac{1}{m !} m[\bar{C}(\beta, \xi)]^{m-1} \\
& =g_{v / 2}\left(\xi e^{\beta b}\right) e^{-\beta b}\|\varrho\| \xi^{q-1} D(\beta) \exp [\bar{C}(\beta, \xi)]
\end{aligned}
$$

so that (3.5) converges uniformly.

It remains to show that $\sum_{\mu_{1}=1}^{\infty} \cdots \sum_{\mu_{n}=1}^{\infty} \int E_{00}^{\mu^{n} \beta}\left(d \tilde{\omega}^{n}\right)\left|\frac{\partial \varrho\left(\hat{\omega}^{n}\right)}{\partial \omega_{\alpha}(0)}\right|$ is convergent. Using (3.5) and (3.8) we see that

$\left|\frac{\delta \varrho\left(\hat{\omega}^{n}\right)}{\delta \omega_{\alpha}(0)}\right| \leqq(q-1) \bar{\Phi} \xi^{q}\|\varrho\|+z \xi^{q-1}\|\varrho\| e^{(q-2) \beta b} \exp [\bar{C}(\beta, \xi)] g_{v / 2}\left(\xi e^{\beta b}\right) D(\beta)$

and

$$
\begin{aligned}
& \sum_{\mu_{1}=1}^{\infty} \ldots \sum_{\mu_{n}=1}^{\infty} \int E_{00}^{\mu^{n} \beta}\left(d \tilde{\omega}^{n}\right)\left|\frac{\delta \varrho\left(\hat{\omega}^{n}\right)}{\delta \omega_{\alpha}(0)}\right| \\
& \quad \leqq \bar{\Phi}\|\varrho\| \sum_{j=1}^{n}\left(\prod_{k=1}^{n} \sum_{\mu_{k}=1}^{\infty} \frac{\xi^{\mu_{k}}}{\mu_{k}^{v / 2} \lambda^{v}}\right)\left(\sum_{\mu_{j}=1}^{\infty} \frac{\xi^{\mu_{j}} \mu_{j}}{\mu_{j}^{v / 2} \lambda^{v}}\right) \\
& \quad-z\|\varrho\|\left(\xi e^{2 \beta b}\right)^{-1} \exp [\bar{C}(\beta, \xi)] g_{v / 2}\left(\xi e^{\beta b}\right) D(\beta) \prod_{k=1}^{n} \sum_{\mu_{k}=1}^{\infty} \frac{\xi^{\mu_{k}} e^{\beta b \mu_{k}}}{\mu_{k}^{v / 2} \lambda^{v}} \\
& \quad=\Phi \frac{\|\varrho\| n}{\lambda^{v n}\left[g_{v / 2}(\xi)\right]^{n-1}\left(g_{v / 2-1}(\xi)\right)} \\
& \quad+z\|\varrho\|\left(\xi e^{2 \beta b}\right)^{-1} \exp [\bar{C}(\beta, \xi)] g_{v / 2}\left(\xi e^{\beta b}\right) D(\beta)\left[g_{v / 2}\left(\xi e^{\beta b}\right)\right]^{n} .
\end{aligned}
$$

This establishes the existence of $\frac{\partial \varrho\left(x^{n} ; y^{n}\right)}{\partial x_{\alpha}}$. 


\section{Similar steps show the existence of second derivatives whenever}

$$
\int\left|\frac{\partial^{2} e^{-\beta \Phi(x)}}{\partial x^{2}}\right| d x<+\infty .
$$

Acknowledgements. Thanks are due to Dr. Gallavotti who introduced me to this subject, to Dr. Ginibre who read preliminary versions and suggested improvements (especially the use of K. S. equations), and to Dr. Lebowitz who suggested this problem and was helpful throughout.

\section{References}

1. Gallavotti, G.: Nuovo Cimento 52b, 208 (1968).

2. Ruelle, D.: Statistical Mechanics. New York: Benjamin 1969.

3. Ginibre, J.: J. Math. Phys. 6, 238 (1965).

4. Ginibre, J.: J. Math. Phys. 6, 252 (1965).

5. Bogoliubov, N. N.: Lectures in quantum statistics, Vol. 1. New York: Gordon \& Breach 1967.

6. Ginibre, J.: Some applications of functional integration in statistical mechanics. Les Houches Summer School Lecture. New York: Gordon \& Breach 1970.

7. Kac, M.: Probability and related topics in physical sciences. London: Interscience 1959.

S. Fesciyan

Belfer Graduate School of Sciences Yeshiva University

New York, N.Y. 10033/USA 